\title{
X Congreso Internacional sobre Investigación en la Didáctica de las Ciencias: una invitación para pensar los desafíos de la educación científica y de la práctica docente
}

Del 5 al 8 de septiembre de 2017 se celebrará el X Congreso de Investigación en Didáctica de las Ciencias, que organiza la revista Enseñanza de las Ciencias.

En esta edición, el Congreso se centra en tres grandes desafíos para la docencia e investigación en nuestra área:

1. ¿Qué educación científica es relevante en el mundo actual? Con dos grandes apartados: ¿Qué educación científica es relevante en un mundo tecnológico? y ¿Qué educación científica es relevante en un mundo en decrecimiento?

2. ¿Qué educación científica es relevante en el contexto científico actual?

3. ¿Cómo reducir la brecha entre investigación y práctica docente?

A esta décima edición se podrán presentar trabajos en la modalidad de simposios y comunicaciones-posters. El plazo de envío empezará el próximo 14 de octubre y finalizará el 18 de diciembre de 2016.

Toda la información de cómo participar, los plazos de inscripción etc., los podéis consultar en la página web www.congresoenseciencias.org, en la que próximamente también estará disponible el programa.

El Congreso estará presente en las redes sociales y puedes seguirlo a través de su perfil en twitter: @CongresoEnsCC y su página en Facebook: https://www.facebook.com/Congresoenseciencias

Esperamos que esta información sea de vuestro interés.

Ana Rivero

Dpto. Didáctica de las Ciencias, Universidad de Sevilla
Rut Jiménez

Dpto. de Educación, Universidad de Almería

Directoras del X Congreso de Investigación en Didáctica de las Ciencias

Encontrarnos con el Congreso número X de Investigación en Didáctica de las Ciencias, suscita una mirada retrospectiva frente a lo que éste ha significado en los últimos 30 años.

Por una parte, el reconocimiento de la revista Enseñanza de las Ciencias (Revista de investigación y experiencias didácticas), organizadora del congreso, y una de las de mayor tradición en la Didáctica de las Ciencias en el habla hispana, cuyo primer número fue editado en 1983. A la fecha, la revista se mantiene como uno de los más relevantes medios de difusión para investigadores y maestros en la enseñanza de las ciencias, ya que en este espacio se han socializado resultados de investigaciones en torno a diferentes problemáticas, enriqueciendo de este modo tanto el desarrollo conceptual como metodológico del área. Actualmente se puede acceder de manera gratuita en el Depósito Digital de Documentos de la Universidad Autónoma de Barcelona, en el siguiente enlace http://ddd.uab.cat/record/1 8? In=carecord/14? ln=ca 
Por otra parte, evidenciamos el tejido de conocimientos construidos a la par entre la revista y los congresos, los cuales se organizaron desde los inicios de la revista. El Primer Congreso, celebrado en el año 1985, incluyó además de la Didáctica de las Ciencias, la de las Matemáticas, así como los tres eventos posteriores. Con el fin de contextualizar grosso modo, los nombres, fechas, lugares y ejes temáticos que se abordaron en estos nueve congresos, los recogeremos en la siguiente tabla.

Tabla 1. Congresos de Investigación en Didáctica de las Ciencias organizados por la Revista Enseñanza de las Ciencias ${ }^{1}$

\begin{tabular}{|c|c|c|}
\hline Nombre Congreso & Fecha y lugar & $\begin{array}{l}\text { Ejes temáticos/número de ponencias } \\
\text { y organización }\end{array}$ \\
\hline $\begin{array}{l}\text { I Congreso Internacional sobre } \\
\text { Investigación en la Didáctica de las } \\
\text { Ciencias y de las Matemáticas }\end{array}$ & $\begin{array}{l}\text { Septiembre } \\
\text { de } 1985, \\
\text { Barcelona }\end{array}$ & 48 ponencias publicadas en el índice \\
\hline $\begin{array}{l}\text { II Congreso Internacional sobre } \\
\text { Investigación en la Didáctica de las } \\
\text { Ciencias y de las Matemáticas }\end{array}$ & $\begin{array}{l}\text { Septiembre de } \\
1987, \text { Valencia }\end{array}$ & $\begin{array}{l}\text { Cerca de } 200 \text { comunicaciones } \\
\text { - Temas generales } \\
\text { - Didáctica de la Biología y de la Geología } \\
\text { - Didáctica de la Física y de la Química } \\
\text { - Didáctica de las Matemáticas }\end{array}$ \\
\hline $\begin{array}{l}\text { III Congreso Internacional sobre } \\
\text { la Didáctica de las Ciencias y de } \\
\text { las Matemáticas. La Investigación } \\
\text { Didáctica y el Trabajo en el Aula }\end{array}$ & $\begin{array}{l}\text { Septiembre de } \\
\text { 1989, Santiago } \\
\text { de Compostela }\end{array}$ & $\begin{array}{l}\text { Cerca de } 300 \text { comunicaciones } \\
\text { - Temas generales } \\
\text { - Didáctica de la Biología y de la Geología } \\
\text { - Didáctica de la Física y de la Química } \\
\text { - Didáctica de las Matemáticas }\end{array}$ \\
\hline $\begin{array}{l}\text { IV Congreso Internacional sobre } \\
\text { Investigación Didáctica de las } \\
\text { Ciencias y de las Matemáticas }\end{array}$ & $\begin{array}{l}\text { Septiembre } \\
\text { de 1993, } \\
\text { Barcelona }\end{array}$ & - Investigaciones didácticas \\
\hline $\begin{array}{l}\text { V Congreso Internacional sobre } \\
\text { Investigación en Didáctica de las } \\
\text { Ciencias. } \\
\text { Investigación e Innovación en la } \\
\text { Didáctica de las Ciencias }\end{array}$ & $\begin{array}{l}\text { Septiembre de } \\
\text { 1997, Murcia }\end{array}$ & $\begin{array}{l}\text { - Formación y desarrollo profesional del } \\
\text { profesorado de ciencias } \\
\text { - Estrategias para la enseñanza-aprendizaje de } \\
\text { las ciencias } \\
\text { - Modelos de desarrollo curricular }\end{array}$ \\
\hline $\begin{array}{l}\text { VI Congreso Internacional sobre } \\
\text { Investigación en la Didáctica de las } \\
\text { Ciencias. } \\
\text { Retos de la enseñanza de las ciencias } \\
\text { en el siglo XXI }\end{array}$ & $\begin{array}{l}\text { Septiembre } \\
\text { de } 2001, \\
\text { Barcelona }\end{array}$ & $\begin{array}{l}\text { Cerca de } 600 \text { aportaciones } \\
\text { - Retos con relación a qué ciencia enseñar } \\
\text { - Retos con relación a cómo enseñar Ciencias } \\
\text { - Retos con relación a cómo formar al } \\
\text { profesorado de ciencias }\end{array}$ \\
\hline
\end{tabular}

1 Elaborada con base en las memorias de los eventos publicadas en los números extraordinarios de la revista Enseñanza de las Ciencias. 


\begin{tabular}{|c|c|c|}
\hline Nombre Congreso & Fecha y lugar & $\begin{array}{l}\text { Ejes temáticos/número de ponencias } \\
\text { y organización }\end{array}$ \\
\hline $\begin{array}{l}\text { VII Congreso Internacional sobre } \\
\text { Investigación en la Didáctica de las } \\
\text { Ciencias. } \\
\text { Educación científica para la } \\
\text { ciudadanía }\end{array}$ & $\begin{array}{l}\text { Septiembre de } \\
\text { 2005, Granada }\end{array}$ & $\begin{array}{l}\text { - Enseñanza de las Ciencias y sociedad } \\
\text { - Proyectos curriculares "en contexto" } \\
\text { la investigación, la innovación y la práctica } \\
\text { - Procesos comunicativos en la enseñanza y } \\
\text { difusión de las Ciencias }\end{array}$ \\
\hline $\begin{array}{l}\text { VIII Congreso Internacional sobre } \\
\text { Investigación en Didáctica de las } \\
\text { Ciencias }\end{array}$ & $\begin{array}{l}\text { Septiembre } \\
\text { de } 2009, \\
\text { Barcelona }\end{array}$ & $\begin{array}{l}\text { Más de } 1000 \text { aportaciones } \\
\text { - Enseñanza de las Ciencias y sociedad } \\
\text { - Ciencias y enseñanza de las Ciencias } \\
\text { - Enseñanza de las Ciencias y currículum } \\
\text { - Enseñanza de las Ciencias en el aula }\end{array}$ \\
\hline $\begin{array}{l}\text { IX Congreso sobre Investigación en } \\
\text { Didáctica de las Ciencias. } \\
\text { La investigación en didáctica de } \\
\text { las ciencias: un compromiso con la } \\
\text { sociedad del conocimiento }\end{array}$ & $\begin{array}{l}\text { Septiembre de } \\
\text { 2013, Girona }\end{array}$ & $\begin{array}{l}\text { Más de } 1.200 \text { aportaciones de las cuales se } \\
\text { seleccionaron } 900 \\
\text { - Educación científica en contextos formales } \\
\text { - Educación científica en contextos no formales } \\
\text { - Educación científica en entornos virtuales } \\
\text { - Formación inicial y permanente de profesorado } \\
\text { - Educación ambiental y Educación para la } \\
\text { sostenibilidad } \\
\text { - Historia y naturaleza de la ciencia } \\
\text { - Aprendizaje científico. Factores cognitivos, } \\
\text { emocionales y sociales } \\
\text { - Lenguaje y argumentación } \\
\text { - Evaluación de programas y aprendizajes } \\
\text { - Enfoques de la educación científica en } \\
\text { contexto } \\
\text { - Complejidad e interdisciplinariedad } \\
\text { - Diseño, implementación y evaluación de } \\
\text { propuestas didácticas } \\
\text { - Educación científica y diversidad }\end{array}$ \\
\hline
\end{tabular}

La panorámica que se vislumbra en la anterior tabla, nos señala, por un lado, la creciente participación, con comunicaciones procedentes de muy diversas nacionalidades; así, por ejemplo, se pasó de cerca de 200 comunicaciones en 1985 a más de 1.200 aportaciones en el último congreso en 2013, con una notoria contribución de investigadores procedentes de América. Por otro lado, destacamos la movilidad que el Congreso ha buscado al descentralizar su organización, permitiendo de este modo, no sólo una mayor participación y apropiación del mismo, sino el reconocimiento de otras perspectivas y dinámicas en su desarrollo.

Respecto de las temáticas que en estos se han presentado, destacamos la constitución de un evento autónomo de las Didácticas de las Matemáticas a partir del V Congreso, por ello en esa quinta versión del Congreso se planteó como tema central la "Investigación e Innovación en la Didáctica de las Ciencias", y uno de los objetivos del evento fue el de "clarificar y profundizar 


\section{Pmotías}

el marco teórico del conocimiento didáctico". Destacamos que el congreso previo fue realizado en el marco de la revista de Enseñanza de las Ciencias, lo que llevó a diferentes balances de la investigación en Didáctica de las Ciencias. También, a partir de este V Congreso se resalta la diversidad de temas y discusiones que han convocado, por ejemplo, sobre: nuevos enfoques del currículo, como CTS y Educación ambiental (V Congreso, 1997); retos para la enseñanza de las ciencias en el siglo XXI (VI Congreso, 2001); complejidad, interdisciplinaridad, modelización, perspectivas socioculturales (VIII Congreso, 2009), entre otros tópicos, que nos permiten relacionar con diferentes perspectivas para comprender los problemas centrales de la Didáctica de las Ciencias.

Esta versión, a la que todos y todas estamos invitados, del X Congreso de Investigación en Didáctica de las Ciencias, propone diferentes líneas, algunas consideradas en anteriores eventos (por ejemplo, Educación científica en contextos formales o no formales) y otras, nuevas (por ejemplo, Progresiones de aprendizaje y modelización). Las líneas para la convocatoria 2017 son: Educación científica en contextos formales en Educación Infantil y Educación Primaria; Educación científica en contextos formales en Educación Secundaria; Educación científica en contextos no formales, informales y virtuales; Educación científica en la universidad; Formación inicial y permanente de profesorado de Educación Infantil y Educación Primaria; Formación inicial y permanente de profesorado de Educación Secundaria y Universidad; Educación Ambiental; Historia y naturaleza de la ciencia; Aprendizaje de las ciencias; Progresiones de aprendizaje y modelización; Lenguaje y argumentación; Educación científica en contexto y controversias sociocientíficas; Interdisciplinariedad, globalización y complejidad; Diseño, implementación y evaluación de programas y propuestas didácticas; Educación científica y género.

Desde estos ejes temáticos, queremos reiterar la invitación a toda la comunidad de maestros e investigadores a participar en este X Congreso, el cual se llevará a cabo en la ciudad de Sevilla (España), entre los días 5 y 8 de septiembre de 2017.

Carmen Alicia Martínez Rivera²

2 Doctorado Interinstitucional en Educación, Universidad Distrital Francisco José de Caldas, camartinezr@udistrital.edu.co 